\title{
Stem Cells in the Treatment of Refractory Chronic Migraines
}

\author{
Alexander Mauskop $^{a} \quad$ Kenneth O. Rothaus ${ }^{b}$ \\ ${ }^{a}$ New York Headache Center, Department of Neurology, SUNY Downstate Medical Center, \\ New York, NY, USA; ${ }^{b}$ Division of Plastic and Reconstructive Surgery, Cornell Medical \\ College, New York, NY, USA
}

\section{Keywords}

Stem cells $\cdot$ Chronic migraine $\cdot$ Refractory headache $\cdot$ Refractory chronic migraine

\begin{abstract}
Background: Autologous adipose-derived stromal vascular fraction (SVF), which is rich in mesenchymal stromal cells, has been reported to be effective for the treatment of trigeminal neuropathic pain and chronic migraine and tension-type headaches. It is possible that stem cell activity targets neurogenic inflammation, which is a well-documented aspect of migraine pathogenesis. Methods: Adult patients with severe migraine-related disability as measured by the Migraine Disability Assessment (MIDAS) score who failed botulinum toxin injections and at least 3 prophylactic drugs were included in this study. The primary outcome measure was the change in MIDAS score 3 months after treatment. Standard liposuction was performed to obtain adipose tissue, from which SVF was isolated by centrifugation. A sample of each patient's SVF was tested for the number of nucleated cells and their viability. Between 8 and $10 \mathrm{~mL}$ of SVF with 2.5-8.6 million viable cells were injected into the pericranial, neck, and trapezius muscles. Results: One man and 8 women were enrolled in the study. The mean age was 48 years, the mean duration of headaches was 16 years, the mean number of prophylactic drugs tried was 10, and the mean MIDAS score at baseline was 122. Three months after the procedure the mean MIDAS score was 88 . Seven out of 9 patients had a decrease in their
\end{abstract}

\section{KARGER}

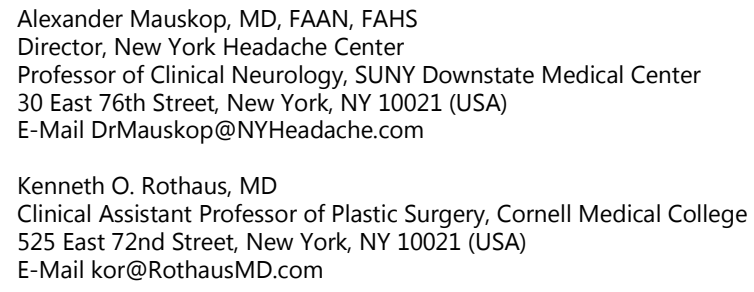


MIDAS score, but only 2 had meaningful improvement. Conclusion: The use of autologous adipose-derived SVF may be effective in the treatment of chronic refractory migraines. It is possible that the use of allogenic stem cells could offer a more practical and more effective approach.

\section{Introduction}

Despite the availability of highly effective abortive and prophylactic therapies for migraines, a significant minority of migraine sufferers do not respond to these treatments. The definition of refractory chronic migraine is still being debated [1], but we do know that this condition affects a relatively small but significant proportion of chronic migraine patients [2].

Facing a large number of patients with migraines who have not responded to a variety of treatments and following the publication of 4 case reports of refractory headaches being successfully treated with stem cells [3], we proceeded to treat some of our most refractory patients with stem cell therapy.

Mesenchymal stem cells, or mesenchymal stromal cells (MSCs), are multipotent cells that are relatively easy to isolate and that have many potential therapeutic applications. In addition to bone marrow, MSC populations can be readily obtained from adipose and a variety of other tissues including placenta, skin, umbilical cord, and others. The mechanism of action of these cells remains to be fully elucidated, but their safety when given intravenously has been fairly well established [4]. There are indications that an intramuscular injection of stem cells provides a longer-lasting effect than an intravenous infusion [5].

Animal studies indicate that MSCs are effective in models of experimental autoimmune encephalomyelitis, traumatic brain injury, and stroke. These effects do not appear to require CNS implantation by MSCs, but result from the inhibition of pathogenic immune responses and release of neuroprotective molecules that promote tissue repair.

Autologous adipose-derived stromal vascular fraction (SVF) is rich in MSCs and has been reported to be effective for the treatment of trigeminal neuropathic pain [6]. It is possible that stem cell activity targets neurogenic inflammation, which is a well-documented aspect of migraine pathogenesis.

\section{Methods}

Women and men over the age of 18 with refractory chronic migraines with or without medication overuse were included. Refractoriness was defined as severe migraine-related disability as measured by the Migraine Disability Assessment (MIDAS) score and failure to respond to at least 3 prophylactic medications from at least 2 different classes and onabotulinumtoxinA. All patients signed an informed consent approved by an independent ethics committee Exclusion criteria were significant abnormal findings on complete blood count or metabolic panel, pregnancy, lactation, current acute systemic illness, serious psychiatric disorder, and lack of sufficient amounts of adipose tissue.

The main outcome measure was change in the MIDAS score 3 months after the treatment (MIDAS score above 20 indicates severe disability). Statistical analysis was planned but not carried out because of the high heterogeneity of patients, including comorbid neurologi- 
cal conditions and continued use of onaboltulinumtoxinA injections and changes of prophylactic medications in some patients.

We also recorded the Headache Impact Test (HIT-6) score, 7-point Patient Global Impression of Change (PGIC), Clinician's Global Impression of Change (CGIC), number of headache-free days, percentage of patients with $50 \%$ or greater improvement in headache-free days, and number and type of abortive medications taken.

A standard mini-liposuction was performed under local anesthesia to obtain 240-360 $\mathrm{mL}$ of adipose tissue, from which SVF was separated by centrifugation. A sample of each patient's SVF was tested for the number of nucleated cells and their viability. Between 8 and $10 \mathrm{~mL}$ of SVF were injected into the temporalis, occipitalis, neck, and trapezius muscles. The injected SVF contained between 2.5 and 8.6 million viable nucleated cells.

\section{Case Reports}

\section{Patient 1}

Patient 1 was a 36-year-old woman with 20 years of daily headaches. Despite her disability, she remained self-employed and got married. Her headaches were partially controlled with onabotulinumtoxinA injections, topiramate $(100 \mathrm{mg})$, nortriptyline $(50 \mathrm{mg})$, and sumatriptan (100-mg tablets taken 3 times a day). Her migraines remained daily despite prolonged periods of abstinence from abortive medications. Her reason for enrolling in the trial was to be able to discontinue topiramate and then to get pregnant.

Within a week of the procedure, the patient reported a dramatic improvement in her headaches, with pain in the mild range instead of moderate or severe. These headaches were controlled with $50 \mathrm{mg}$ of sumatriptan. One month after the procedure her HIT-6 score dropped from 65 at baseline (severe impact) to 46 (little or no impact), and she lowered her topiramate to $75 \mathrm{mg}$ and then to $50 \mathrm{mg}$, with worsening of her headaches to pretreatment level. She increased topiramate back to $100 \mathrm{mg}$ and remained on nortriptyline, onabotulinumtoxinA, and sumatriptan (100 mg 3 times a day).

She had only minimal improvement on the PGIC scale at the end of the study. Her MIDAS score dropped from 150 at the start to 105 at 3 months. Her HIT-6 score was 65 at baseline and 66 in the third month.

\section{Patient 2}

Patient 2 was a 44-year-old woman with migraine headaches since the age of 8 , which became daily at the age of 41 , following a mild concussion. She had failed 6 prophylactic medications, but continued to receive onabotulinumtoxinA injections and monthly infusions of magnesium throughout the study. She took eletriptan $(40 \mathrm{mg}$ ) on 22 out of 30 days in the month before the study. The patient was married with 3 adult children, lived with her husband and worked full time as the president of her own company.

Her MIDAS score dropped from 210 to 84 and her HIT-6 score from 76 to 66 in the third month, and she reported minimal improvement from the procedure on the PGIC scale. There was no change in her intake of abortive medications

\section{Patient 3}

Patient 3 was a 49-year-old woman with headaches since the age of 7, which became very frequent at the age of 45 . She had tried 6 prophylactic drugs and remained on $5 \mathrm{mg}$ of nebivolol and $150 \mathrm{mg}$ of venlafaxine. For abortive therapy, she took injectable sumatriptan 
(6 mg) or injectable ketorolac (60 mg) on average once a week. She was working full-time as a psychotherapist and lived with her husband and 10-year-old son.

After the SVF procedure her MIDAS score dropped from 45 to 22 and her HIT-6 score from 66 to 57 in the third month. She did not take any injectable abortive medications throughout the 3-month period and took only acetaminophen or ibuprofen on 11 out of 90 days. She had 27 headache-free days in the third month after the procedure compared to 15 at baseline. The PGIC scale was rated as very much improved.

\section{Patient 4}

Patient 4 was a 61-year-old woman with a 32-year history of chronic migraines and had been retired for 5 years due to migraine-related disability. She had tried 20 prophylactic medications with no relief and was taking naratriptan daily as well as oxycodone (5 mg) with $325 \mathrm{mg}$ of acetaminophen on not more than 6 days a month. On another 5-6 days a month she was taking intranasal ketorolac. She had 3 hospitalizations for the treatment of medication overuse. She also suffered from asthma (controlled by medications), hypertension (treated with olmesartan and amlodipine), fibromyalgia (relieved by $60 \mathrm{mg}$ duloxetine), lupus (in remission), insomnia (treated with zolpidem), and hypothyroidism (treated with $112 \mu \mathrm{g}$ levothyroxine). She was happily married with adult children.

The patient felt somewhat worse in the month after the SVF procedure, although her HIT-6 score remained unchanged at 66 . An increase of duloxetine from 60 to $120 \mathrm{mg}$ a day brought her migraines back to the pretreatment baseline. After 3 months she reported minimal improvement on the PGIC scale with her MIDAS score dropping from 79 to 62, although the dose of duloxetine was increased during this period. She had no headache-free days before or after the treatment, and there was no change in her intake of abortive medications.

\section{Patient 5}

Patient 5 was a 53-year-old man with a 23-year history of headaches. At the age of 34 and after a year of persistent headaches he was found to have hydrocephalus which was treated with a ventriculoperitoneal shunt. The shunt reduced the size of his ventricles but did not improve headaches, and he had to stop working. He had tried 15 prophylactic drugs without any relief. At the start of the study he was on $60 \mathrm{mg}$ of duloxetine for depression and $150 \mathrm{mg}$ of armodafinil for fatigue. He also had osteoarthritis and had had bilateral knee surgeries, a hip replacement, and cervical spine discectomies. He had been taking an average of 5 tablets of $5 \mathrm{mg}$ oxycodone with $325 \mathrm{mg}$ of acetaminophen each month for his joint pains, but no abortive drugs for his headaches. His marriage ended in a divorce at the age of 43 . He owned a house in Hawaii and had been able to regularly travel there from New Jersey.

He reported minimal improvement on the PGIC scale after the SVF procedure with his MIDAS score dropping from 220 to 160 and his HIT-6 score dropping from 74 at baseline to 66 after 3 months. He had no headache-fee days before or after the procedure. He took no abortive drugs before or after the procedure.

\section{Patient 6}

Patient 6 was a 48-year-old woman with 25 years of headaches. Her chronic migraines responded to onabotulinumtoxinA for about 10 years, but in the preceding 3 years the response diminished and her disability became severe. She had tried over 20 prophylactic medications with no relief, but continued her onabotulinumtoxinA injections and monthly intravenous magnesium infusions. Abortive medications provided some relief and she had been taking zolmitriptan, alternating with eletriptan, tramadol, ibuprofen, and, very occa- 
sionally, butalbital/caffeine/acetaminophen. She was a practicing attorney and was happily married with 3 children.

Three months after the SVF injections she reported feeling much improved on PGIC scale, with her MIDAS score dropping from 44 to 15 and her HIT-6 score dropping from 66 at baseline to 63 in the third month. She had no change in headache-free days - 10 before and 10 after the procedure. The number and type of abortive medications also did not change.

\section{Patient 7}

Patient 7 was a 53-year-old woman with 30 years of chronic migraine headaches. The frequency of her migraines progressed from 2-3 a week on her first visit to daily headaches 5 years later. She had failed many nondrug therapies, nerve blocks, and 15 prophylactic medications. Eletriptan and rizatriptan provided partial and temporary relief and she took them on an average twice a week. Triptan-free periods of up to 2 weeks did not provide any relief. She was otherwise in very good health and was happily married with 2 children. She worked as a dentist, but had to stop due to migraines 3 years before this treatment.

She reported no change in her headaches on the PGIC scale 3 months after the procedure. Her MIDAS score dropped from 90 to 84 and her HIT-6 score remained unchanged at 69 in the last month. She had 6 headache-free days in the month before treatment and 5 in the third month after treatment. There was no change in the number or the type of abortive medications taken.

\section{Patient 8}

Patient 8 was a 56-year-old woman with 5 years of migraine, which became chronic 3 years earlier when she went through menopause. She had tried 4 prophylactic drugs and all caused side effects. OnabotulinumtoxinA injections were partially effective and she continued receiving them throughout the study. She also continued to receive monthly infusions of magnesium. She was happily married and working full-time as a guidance counselor.

One month after the SVF injections her HIT-6 score dropped from 57 to 48, and the PGIC scale was rated as much improved. Two months later her HIT-6 score was 54 and the PGIC scale was still rated as much improved. Early into the third month she had a menstrual period after not having had any for 5 months, and her headaches dramatically worsened. Three months after the procedure, her HIT-6 score was 52 and her MIDAS score 45 - up from 36 on day 0 . The PGIC scale at 3 months was minimally worse. She had no headache-free days, and her daily intake of sumatriptan did not change.

\section{Patient 9}

Patient 9 was a 34-year-old woman with migraine headaches of 21 years in duration, which became daily and severe at the age of 28 . At 29 she was found to have increased intracranial pressure with small ventricles and had a ventriculoperitoneal shunt placed. The shunt did not relieve her headaches. She also suffered from hypothyroidism, WolffParkinson-White syndrome, interstitial cystitis, Crohn's colitis, and polycystic ovarian syndrome. She had tried more than 12 prophylactic medications. For abortive therapy, she was taking $2.5 \mathrm{mg}$ of frovatriptan and $40 \mathrm{mg}$ of eletriptan daily, and either diclofenac $(50 \mathrm{mg})$ or tramadol $(50 \mathrm{mg})$ on more than half of the days. The patient had a postgraduate degree in public health but had been unable to work due to headache. She was married with a very supportive husband and parents. 
The SVF injections had no effect on her headaches - her MIDAS score remained 180 and the HIT- 6 score was 72 before and 68 after the treatment. She had no headache-free days before or after the procedure, and her triptan and analgesic intake did not change.

\section{Results}

One male and 8 female patients were enrolled in the study. The mean age was 48 years, the mean duration of headaches was 16 years, and the mean number of prophylactic drugs tried was 10. Two patients had functioning ventriculoperitoneal shunts. Three months after the procedure the MIDAS score dropped in 7 out of 9 patients. The PGIC scale was reported as 1 very much improved, 1 much improved, 4 minimally improved, 2 no change, and 1 minimally worse. One of the patients reporting minimal improvement had a dramatic improvement within the first month and until she lowered the dose of her topiramate. Another patient was much improved for 2 months after the procedure, but worsened with the onset of menstruation, after not having menstruations for 5 months. The number of headache-free days, the percentage of patients with $50 \%$ or greater improvement in headache-free days, and the number and type of abortive medications taken showed no change. No adverse events were observed.

\section{Discussion}

These case reports of patients afflicted with refractory chronic migraines suggest that some such patients may improve with stem cell therapy. Stem cells may relieve migraines through their proven anti-inflammatory properties because neurogenic inflammation is one of the major aspects of migraine pathogenesis. A decrease in the number and function of endothelial progenitor cells has been found in patients with migraine [7], and this is speculated to be the cause of the increased cerebrovascular risk in migraine patients, while endothelial progenitor cells have been successfully derived from adipose tissue and used for the treatment of traumatic brain injury [8]. Another related finding is that stromal cell-derived factor 1-alpha is decreased in women with migraine with aura [9].

Limitations of this study include its open-label design, the small number of patients, the allowance of concomitant therapies, and changes in those therapies during the study. In addition, 2 patients had ventriculoperitoneal shunts. Considering that these patients were refractory to multiple therapies, the reduction of disability in 7 out of 9 patients, with 2 patients reporting meaningful improvement indicates a possible therapeutic signal.

Because of the difficulty in obtaining autologous adipose-derived stem cells, the more practical approach in future studies may be the use of allogenic adipose, bone marrow, or placenta-derived stem cells. Placenta-derived stem cells avoid the ethical issues of fetal cells and have a more favorable profile than umbilical cord-derived cells [10]. Such cells, once they become commercially available, are likely to have more consistent and well-defined properties. 


\section{Conclusion}

Stem cell therapy has theoretical underpinnings which suggest possible efficacy in the treatment of migraine headaches. Anecdotal evidence suggests that controlled clinical trials are warranted.

\section{Statement of Ethics}

The study was approved by an ethics committee (New England IRB, ref. No. 15-236). All the participants gave their written informed consent before taking part in the study.

\section{Disclosure Statement}

The trial was partly funded by MicroAire Surgical Instruments.

\section{Author Contributions}

A.M.: patient enrollment, treatment injections, data collection, and manuscript preparation. K.O.R.: adipose tissue harvesting and processing, and manuscript review.

\section{References}

1 Martelletti P, Katsarava Z, Lampl C, Magis D, Bendtsen L, Negro A, Bjørn Russell M, Mitsikostas D-DD, Jensen RH: Refractory chronic migraine: a consensus statement on clinical definition from the European Headache Federation. J Headache Pain 2014;15:47.

2 Irimia P, Palma J-A, Fernandez-Torron R, Martinez-Vila E: Refractory migraine in a headache clinic population. BMC Neurol 2011;11:94.

-3 Bright R, Bright M, Bright P, Hayne S, Thomas WD: Migraine and tension-type headache treated with stromal vascular fraction: a case series. J Med Case Rep 2014;8:237.

- 4 Ra JC, Shin S, Kim SH, Kang SK, Kang BC, Lee HY, Kim YJ, Jo JY, Yoon EJ, Choi HJ, Kwon E: Safety of intravenous infusion of human adipose tissue-derived mesenchymal stem cells in animals and humans. Stem Cells Dev 2011;20:1297-1308.

-5 Fischer UM, Harting MT, Jimenez F, Monzon-Posadas WO, Xue H, Savitz SI, Laine GA, Cox CS: Pulmonary passage is a major obstacle for intravenous stem cell delivery: the pulmonary first-pass effect. Stem Cells Dev 2009;18:683-692.

6 Vickers ER, Karsten E, Flood J, et al: A preliminary report on stem cell therapy for neuropathic pain in humans. J Pain Res 2014;7:255-263.

7 Lee ST, Chu K, Jung KH, Kim DH, Kim EH, Choe VN, Kim JH, Im WS, Kang L, Park JE, Park HJ, Park HK, Song EC, Lee SK, Kim M, Roh JK: Decreased number and function of endothelial progenitor cells in patients with migraine. Neurology 2008;70:1510-1517.

-8 Xue S, Zhang HT, Zhang P, Luo J, Chen ZZ, Jang XD, Xu RX: Functional endothelial progenitor cells derived from adipose tissue show beneficial effect on cell therapy of traumatic brain injury. Neurosci Lett 2010;473:186-191.

-9 Liman TG, Neeb L, Rosinski J, Reuter U, Endres M: Stromal cell-derived factor-1 alpha is decreased in women with migraine with aura. Headache 2016;56:1274-1279.

10 Talwadekar MD, Kale VP, Limaye LS: Placenta-derived mesenchymal stem cells possess better immunoregulatory properties compared to their cord-derived counterparts - a paired sample study. Sci Rep 2015;5:15784. 